\title{
Current Role of Minimally Invasive Surgical Techniques in the Diagnosis and Management of Endometriosis-A Clinical Review
}

\author{
Hany Wisa ${ }^{1 *}$, Aiman Aref ${ }^{1}$ and A I Hasib Ahmed ${ }^{1,2}$ \\ ${ }^{1}$ Medway NHS Foundation Trust, Kent, UK \\ ${ }^{2}$ Canterbury Christ Church University, Kent, UK
}

*Corresponding author: Hany wisa, Medway NHS Foundation Trust, Kent, UK.

To Cite This Article: Hany Wisa, Aiman Aref, A I Hasib Ahmed. Current Role of Minimally Invasive Surgical Techniques in the Diagnosis and Management of Endometriosis-A Clinical Review. 2020 - 9(2). AJBSR.MS.ID.001367. DOI: 10.34297/AJBSR.2020.09.001367.

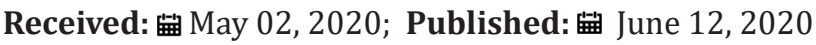

\begin{abstract}
Background: Endometriosis is a very common gynaecological condition associated with chronic pelvic pain and a negative impact on fertility. Various medical and surgical methods have been described to treat endometriosis with varying degrees of success.

Aim: This review examines the evidence behind the current practice of minimal invasive surgery in the diagnosis and management of this condition.

Methods: The Cochrane database was searched for systematic reviews about endometriosis. The ESHRE guidelines were also included. A search of the British Journal of Obstetrics and Gynaecology "BJOG", and the Journal of Minimal Invasive Gynaecology "JMIG" individual database for articles about surgical management of endometriosis was done.

Findings: Diagnostic laparoscopy is the method of choice for diagnosis and is best combined with histological diagnosis. Surgical treatment of endometriosis is superior to medical treatment in terms of recurrence of symptoms and complete cure of the disease. Medical treatment has no role in fertility related endometriosis. Laparoscopic surgery in general is preferred to the open approach. Conservative surgery includes ablation or excision of the peritoneal lesions. Ovarian endometriomas are best treated with excision of the cyst wall rather than ablation. Laparoscopic uterine nerve ablation (LUNA) is not effective in the treatment of endometriosis related pain. Presacral neurectomy (PSN) is effective but requires specific surgical expertise. Deep infiltrating endometriosis affecting the rectum and sigmoid is mainly treated with resection and anastomosis. There is still not enough evidence to support the use of robotic surgery.
\end{abstract}

Conclusion: Minimally invasive surgical techniques have a well-established role in the diagnosis and treatment of pain related and infertility related endometriosis. Surgical management is superior to medical management. More trials are awaited to identify the role of robotic surgery in this field.

Keywords: Endometriosis, Endometrioma, Infertility, Laparoscopy, LUNA, PSN, Robotic surgery, Endometriomas, Ovaries

Abbreviations: ESHRE: European Society of Human Reproduction and Embryology; LUNA: Laparoscopic uterine nerve ablation; PSN: Presacral neurectomy

\section{Introduction}

Endometriosis is a very common gynaecological condition associated with chronic pelvic pain and a negative impact on fertility. Various medical and surgical methods have been described to treat endometriosis with varying degrees of success. This essay examines the evidence behind the current practice of minimal invasive surgery in the diagnosis and management of this condition and explores the recent advances in that field. 


\section{Background}

Endometriosis is characterised by the presence of tissue similar in structure and function to endometrium but present outside the uterus, most commonly in the pelvic cavity. The lesions are mainly scattered and implanted into the visceral peritoneal surfaces in the pelvis. The presence of these lesions causes chronic pelvic pain, painful periods, painful sexual intercourse and infertility [1].

The term "Pelvic endometriosis" is used to define implants that involve the pelvic peritoneum including the anterior cul-de-sac (utero-vesical pouch), posterior cul-de-sac pouch of Douglas), pelvic side walls and surface of uterus, tubes. Ovarian endometriosis can present as blood filled cysts known as endometriomas. "Extra-pelvic endometriosis is defined as endometriotic-like implants elsewhere in the peritoneal cavity or other body cavities. Endometriotic implants can involve the bladder, pelvic ureter, sigmoid colon, rectum, ileocaecal area and appendix [2]. Bladder endometriosis can present with cyclical haematuria, dysuria, urgency and frequency [3]. Ureteric involvement can end in ureteric obstruction. Gastrointestinal endometriosis can present with disturbed bowel function and cyclical rectal bleeding. Less commonly endometriosis may be present in the pleural cavity causing cyclical haemoptysis [4].

Endometriosis is present in about $6-10 \%$ of all women of reproductive age. Its prevalence can be as high as 35-50\% in women presenting with pelvic pain, infertility or both [5]. A multicentric cross-sectional prospective study demonstrated significant impairment of the quality of life and work productivity across countries and ethnicities in women suffering from endometriosis [6]. A significant number of women suffering from pain due to endometriosis also show symptoms of depression and anxiety [7].

The negative impact of endometriosis on fertility has been attributed mainly to four mechanisms. Distorted adnexal anatomy due to scarring inhibits the capturing the mature ovum by the fimbrial end of the fallopian tube. Inflammatory changes in the peritoneal fluid can inhibit fertilization of the ovum or interfere with oocyte development and early embryogenesis. Finally, reduced endometrial receptivity impairs the implantation process [8]. Several European studies have shown significant delay in the diagnosis of endometriosis. This could be explained by several factors including wrong diagnosis and the acceptance of menstrual period related pain as a normal phenomenon by patients and doctors [9]. The correct diagnosis can be delayed by up to 8 years in the UK [10].

A wide range of medical and surgical approaches have been practiced for the management of endometriosis. Medical treatment that aims to supress ovulation with hormonal agents is effective in reducing pain in $80-90 \%$ of women; However, pain recurrence is frequent after stopping treatment. Laparoscopy is now considered the preferred management choice for endometriosis owing to the advances in minimal invasive surgery technology and the limitations of medical treatment [11].

\section{Methods}

The Cochrane database was searched for systematic reviews about endometriosis. 6 Cochrane systematic reviews were identified, 5 of which looked at surgical treatment of endometriosis and these were included in this essay. The ESHRE guidelines were included in the review. Some references from the ESHRE were further looked at based on their relevance to the title of the essay and date of publication with preference to the last 5 years. A search of the British Journal of Obstetrics and Gynaecology "BJOG", and the Journal of Minimal Invasive Gynaecology "JMIG" individual database for articles about surgical management of endometriosis was done.

\section{Results}

\section{Laparoscopic diagnosis of endometriosis}

The current guideline of the European Society of Human Reproduction and Embryology (ESHREE) for the management of women with endometriosis, recommends that diagnostic laparoscopy should not be attempted merely for the diagnosis of superficial peritoneal disease if signs of deep infiltrating disease or ovarian endometriosis (endometriomas) were not present on physical examination or pelvic ultrasound imaging. Treatment of peritoneal disease has not been shown to alter the natural course of the disease. Diagnostic laparoscopy for endometriosis should be done with histology for the lesions to confirm the diagnosis and to exclude the rare possibility of malignancy [12].

A systematic review of literature was conducted to evaluate the accuracy of laparoscopy in the diagnosis of endometriosis [13]. The authors searched the literature for studies where diagnosis of endometriosis was verified by histology. Initially 1426 studies were identified but only 27 were included in the review after excluding studies with inappropriate population selection, inappropriate intervention or failed procedures. Duplicate work lacking original data was also excluded. Only 4 studies out of these 27 were controlled studies. However, out of these 4 studies only 2 were blinded and only one mentioned the method of data collection. In addition to highlighting the paucity of high quality studies in this aspect, the authors concluded that a negative diagnostic laparoscopy was highly accurate for excluding the disease and a positive laparoscopy without taking biopsies for histology was of limited value.

\section{Laparoscopic treatment of endometriosis}

Laparoscopic techniques for the treatment of endometriosis depend on the location of the lesions, the severity of symptoms and the aim of the treatment. Conservative surgery includes excision or ablation (destruction by electro-cautery, or laser) of small 
lesions limited to the peritoneal surfaces in addition to division of adhesions. Endometriomas of the ovaries have been treated with either excision of the cyst capsule or drainage and electrocoagulation of the cyst wall. Pelvic denervation to treat endometriosis related pain has also been described. The most common procedures are the laparoscopic uterine nerve ablation (LUNA) and presacral neurectomy (PSN). When preservation of fertility is not an issue, the severity of symptoms can warrant removal of the uterus and both tubes and ovaries as the definitive cure for endometriosis. Classically, abdominal hysterectomy was the normal approach but recent advances in Minimal invasive surgery technology and techniques have made Laparoscopic Hysterectomy the preferred method for radical treatment of Endometriosis [11].

In a Cochrane systematic review of randomised controlled trials (RCTs) to assess the effectiveness and safety of laparoscopic surgery in the treatment of pain and subfertility associated with endometriosis examined ten RCTs with a total of 973 patients [14]. The quality of evidence was evaluated using GRADE methods.

Laparoscopic surgery was associated with decreased overall pain compared with diagnostic laparoscopy alone, both at six months and at 12 months. Compared with diagnostic laparoscopy, laparoscopic surgery was also associated with an increased live birth or ongoing pregnancy rate and increased clinical pregnancy rate. Only two studies collected data on adverse events and reported no events in either arm. Other studies did not report this outcome which limits the value of this review regarding the safety of laparoscopic surgery.

When laparoscopic ablation was compared with diagnostic laparoscopy plus medical therapy (GnRH analogue plus add-back therapy), more women in the ablation group reported that they were pain free at 12 months. Common limitations in the primary studies included lack of clear descriptions of randomisation, blinding and incomplete outcome data. The authors confirmed difficulties in meta-analysis of data due to heterogeneity of the outcome measures used in the trials, which made it difficult to draw clinically relevant conclusions.

Based on this review, both laparoscopic ablation and excision of lesions seem to be similarly effective in the treatment of pain. However, this is based on one small randomised trial of 24 participants that did not report blinding [15] and a larger RCT of 178 participants that lacked explanation of how ablation or excision were done or which method was used to treat ovarian endometriomas [16]. An RCT showed that laparoscopic excision of endometriosis lesions was more effective than placebo (80\% vs $32 \%$ ) in terms of reducing pain and improving quality of life [17]. However this study lacked power with only 19 women in the placebo arm vs 20 women in the treated arm. The ESHREE guideline for the management of endometriosis recommends excision of endometriotic lesions rather than ablation to obtain histological diagnosis. Furthermore, ablation might not be sufficient to deal with endometriotic lesions deeply infiltrating the visceral peritoneum [12].

\section{Pelvic denervation}

Laparoscopic uterine nerve ablation (LUNA) involves making an incision in each uterosacral ligament. This divides the sensory nerve fibres to the uterus and the cervix. This is considered to be a simple procedure that can be performed by most laparoscopic surgeons. In contrast, presacral neurectomy (PSN) is associated with significant morbidity as it involves division of the hypogastric plexus of nerves at the promontory of sacrum which is in close proximity to major blood vessels, therefore requires higher level of skills [18].

A Cochrane review looked at the effectiveness of pelvic denervation for treatment of primary and secondary dysmenorrhea. Six out of the eleven RCTs identified addressed the effectiveness of the treatment for dysmenorrhea secondary to endometriosis. Three out of these trials examined the benefit of LUNA plus conservative laparoscopic surgery (i.e. ablation and or excision) for endometriosis and three trials examined PSN (one trial examined the laparoscopic approach and two trials the open approach). There was no significant risk of bias in the included studies. LUNA combined with surgical treatment of endometriosis lesions did not show to be of any additional benefit for pain relief compared to surgical treatment alone. Laparoscopic PSN combined with surgical treatment of endometriosis was of significant benefit in terms of pain relief compared to surgical treatment alone but was associated with increased risk of adverse events like bleeding, constipation and urinary urgency. Laparoscopic PSN was also shown to be more specific to midline pain. There was insufficient evidence to recommend the use of LUNA for the treatment of dysmenorrhoea regardless of the cause and therefore laparoscopic conservative surgery for endometriosis associated pain should not be combined with LUNA. Laparoscopic PSN is of benefit in the treatment of pain related to endometriosis but for midline pain only and requires very precise surgical techniques due to the potentially hazardous surgery [19].

\section{Treatment of endometriomas}

The pathogenesis of endometriomas is unclear. One possible explanation is the transplantation theory [20] that deposits of endometrium cause adherence between the ovary and the pelvic peritoneum with progressive invagination of the ovary [21]. According to this explanation, the endometrioma should be a pseudocyst and its wall is the inverted ovarian cortex. Surgical treatment of endometriomas has been shown to be superior to medical treatment [22] and when compared to laparotomy, operative laparoscopy is the method of choice as it has been shown to be associated with shorter hospital stay, faster recovery, less costs and 
lower incidence of developing new adhesions [23]. Laparoscopic treatment of ovarian endometriomas can either be done by excision of the cyst capsule or drainage and electrocoagulation of the cyst wall.

A Cochrane systematic review compared the two techniques to determine the most effective way for treatment. Two RCTs were identified comparing laparoscopic excision of the cyst wall with drainage and ablation by bipolar diathermy. The primary outcome was to measure the improvement in pain symptoms and infertility. The secondary outcome was to measure the recurrence of endometriomas and recurrence of symptoms. In both studies, randomisation and allocation of concealment were free of bias. Although the studies were unblended all patients were followed up with no selective outcome reporting. Excision of the cyst was associated with less recurrence of the endometrioma, less painful periods, less painful intercourse, less non-menstrual pelvic pain and subsequent increased spontaneous pregnancy rate in women previously known to be subfertile. There was insufficient evidence as to which technique was better with regards to fertility [24]. A more recent prospective randomized trial evaluated excision of ovarian endometriomas against laser vaporization with a five year follow up for recurrence. The authors reported significantly higher recurrence rate of endometriomas on ultrasound in the group treated with laser vaporization at 12 months compared with the excision group. There was no statistically significant difference of recurrence rate at 5 years follow up [25]. This study was limited by the number of patients allocated to each group (36 for cystectomy and 38 for laser vaporization). According to the authors a sample size of 312 patients per group would be necessary to provide a statistical power of $80 \%$.

\section{Deeply infiltrating endometriosis}

Surgical treatment options for colorectal deep endometriosis include superficial shaving (superficial peeling of bowel serosal and subserosal endometriosis), discoid resection (selective excision of the endometriosis lesion with opening, then closure, of the bowel wall) and segmental resection of the bowel [26]. Donnez and Squifflet [27] debated the evidence for better improvement with the bowel resection technique against the shaving technique. They analysed the complication rate of 500 patients treated by the shaving technique for deep rectovaginal endometriotic nodules. Their findings showed overall all complication rate with the shaving technique (1.4\% rectal perforation, $0.8 \%$ ureteric injury and $0.2 \%$ significant blood loss) and a combined (both spontaneous and IVF) post treatment pregnancy rate of $84 \%$. Their argument is that endometriosis "is not cancer" and therefore does not require the same treatment approach. Accordingly, they support debulking surgery (shaving) against radical surgery (rectal resection). However, this study lacked a control group.
A systematic review concluded that segmental bowel resection for colorectal endometriosis was followed by excellent pain relief for the first year after surgery [28]. Recurrence of pain requiring intervention was reported in 61 out of 314 women. Bowel resection seemed to be a widely acceptable option possibly because bowel surgeons are used to resections for the treatment of bowel cancer. This review did not specify the type of studies included and highlighted the poor reporting of operative techniques, lesion size, or indication for surgery among the studies. In addition, endometriosis was not always confirmed by histology.

There is lack of clear comparison of the clinical outcome of various surgical techniques for the treatment of deep colorectal endometriosis. A systematic review examined 49 studies. $71 \%$ of the patients were treated with bowel resection and primary anastomosis, $10 \%$ with full thickness disc excision and $17 \%$ were treated with superficial surgery [26]. Because of the inconsistency of reporting data throughout the reviewed papers, comparison of clinical outcome between different surgical techniques was not possible. The authors suggested a checklist to standardise the reports of trials for surgical treatment of deep infiltrating endometriosis.

In an RCT that compared laparoscopically assisted versus open colorectal resection for deep bowel endometriosis, 52 women with colorectal endometriosis were randomized with 26 patients in each group. The study showed that laparoscopic colorectal resection was as effective as open technique in terms of improvement in symptoms and quality of life. Laparoscopy was associated with higher spontaneous pregnancy rate compared to open surgery [29]. Although the authors stated that "this study was not designed to test whether laparoscopic assisted colorectal resection is superior to open surgery for endometriosis", they reported that laparoscopy required less postoperative analgesia than open surgery and is a safe option with overall less severe complications in the laparoscopy group (11 versus 25). There was a 7.7\% conversion rate from laparoscopic to open surgery. This study lacked power due to small number of patients in each arm and was unblinded.

\section{Laparoscopic Hysterectomy}

Hysterectomy with removal of tubes and ovaries has long been proposed as the definitive cure for endometriosis. Classically, the operation was done abdominally. Recent technological advances in minimally invasive surgery has led to the emergence of the laparoscopic approach as the preferred method owing to less postoperative pain and faster recovery. Evidence to support hysterectomy as the definitive treatment for pain related to endometriosis remains controversial. In a non-systematic review of literature [30], 80 references relative to laparoscopic hysterectomy for endometriosis were identified. The author identified 80 
references relevant to the subject and concluded that hysterectomy significantly improved non-specific pelvic pain associated with endometriosis. However, there was of differentiation in the literature reviewed between cyclical and non-cyclical pelvic pain, making interpretation of the findings difficult. Hysterectomy with conservation of ovaries was associated with a six fold increased risk for recurrent pelvic pain and eight times increased risk for needing further surgery compared to hysterectomy with removal of the ovaries. The Martin review [30] reiterates controversy in considering total abdominal hysterectomy and bilateral salpingoophorectomy (TAH-BSO) as "definitive surgery" for endometriosis. The term "definitive surgery" implies that TAH-BSO results in complete cure of pain. The assumption is that removing the ovaries will result in regression of any remaining endometriotic deposits due to removal of estrogenic stimulus. However, requirement for further surgery for endometriosis after TAH-BSO has been described. Radical excisional surgery is more appropriately termed "definitive cure" where in addition to TAH-BSO, all indefinable endometriotic lesions are excised including lesions on the bowel, bladder and ureter.

The laparoscopic approach to hysterectomy as a treatment for severe pelvic endometriosis has been compared to the abdominal approach in a retrospective analysis of 503 patients who underwent hysterectomy for severe endometriosis [31]. 115 patients underwent laparoscopic hysterectomy and 388 abdominal hysterectomies. Measured surgical outcomes were operative time, blood loss, need for blood transfusion and duration of hospital stay. The results were in favour of laparoscopic hysterectomy in terms of the last three parameters but operative time was significantly lower with the abdominal approach. There was a $4.3 \%$ conversion rate from laparoscopic to abdominal approach. With lower complication rates in those women treated by the laparoscopic approach, it is reasonable to consider laparoscopy as the approach of choice for patients undergoing hysterectomy for endometriosis. This recommendation needs confirmation by prospective RCTs.

\section{Robotic Hysterectomy}

A systematic review in 2011 identified only 4 published articles about the use of robotic assisted laparoscopic surgery for endometriosis [32]. Three studies were case reports, one of which is detailed below, and the fourth was a cohort study discussed further. All the studies showed that robotic surgery seems to be safe with no report of complications. No randomized controlled trials exist on this subject.

A large retrospective cohort study compared robotic-assisted laparoscopy with conventional laparoscopy for treatment of advanced stage endometriosis [33]. Measured parameters included operative time, estimated blood loss, complication rate, and length of hospital stay. 273 patients underwent conventional laparoscopy and 147 underwent robotic assisted surgery. There were no significant differences in blood loss or complication rate between the 2 groups but the mean operative time was more in the robotic assisted group (196 minutes) compared to the conventional laparoscopy group (135 minutes). Length of hospital stay was also significantly increased in the robotic-assisted laparoscopy group. The longer duration of the robotic technique was related to several factors. Dealing with large specimens like ovarian endometrioma was more difficult with the robotic method for technical reasons. Also the use of $\mathrm{CO} 2$ laser for ablation of lesions is not possible with the robotic method and more time and care is required to achieve the ablation with robotic scissors and monopolar hook. Also the limited flexibility in changing the camera places in the robotic technique contributed to longer operative time.

Currently no papers exist in the literature to compare laparoscopic hysterectomy with robotic hysterectomy specifically for endometriosis; however, several studies have compared the two techniques for hysterectomy for differentindications. Aretrospective study comparing total laparoscopic hysterectomy versus robotic hysterectomy in terms of operative time, complications and hospital stay does not support the widespread use of robot for hysterectomy [34]. 77 patients underwent total laparoscopic hysterectomies and 47 patients underwent robotic hysterectomies. The authors found no justification to favour the routine use of robotic assisted technique for hysterectomy compared to conventional laparoscopy. The results of this study lack generalisation as only two surgeons performed all the operations included. Robotic technique might be a way for less experienced surgeons to change faster to more minimally invasive approach to gynaecologic surgery, however, with surgeons skilled in conventional laparoscopic hysterectomy, the robot rarely improves the outcome.

\section{Conclusion}

Endometriosis is a very common gynaecological condition characterised by the presence of ectopic endometrial tissue outside the uterus that causes chronic pelvic pain, pelvic adhesions and impaired fertility. It can adversely affect the quality of life of the sufferer and may cause depression and anxiety. Up to $50 \%$ of women suffering from pelvic pain are found to have endometriosis. Diagnostic laparoscopy is the method of choice for diagnosis and is best combined with histological diagnosis.

Surgical treatment of endometriosis is superior to medical treatment in terms of recurrence of symptoms and complete cure of the disease. Medical treatment has no role in fertility related endometriosis. Laparoscopic surgery in general is preferred to the open approach due to fewer complications, less postoperative pain, shorter hospital stay and faster recovery. 
Conservative surgery which aims to spare fertility includes ablation or excision of the peritoneal lesions with comparable outcomes. Excision adds the benefit of histological diagnosis so it is the recommended method for mild to moderate disease. Ovarian endometriomas are best treated with excision of the cyst wall rather than ablation to avoid recurrence. Laparoscopic uterine nerve ablation (LUNA) is not effective in the treatment of endometriosis related pain. Presacral neurectomy (PSN) is effective but requires specific surgical expertise owing to the higher risk of complications and higher morbidity. Deep infiltrating endometriosis affecting the rectum and sigmoid is mainly treated with resection and anastomosis. There is insufficient evidence to support such radical surgery compared to shaving or excision of the lesions without bowel resection. Definitive surgical treatment of endometriosis requires radical excision of all identifiable endometriotic deposits. Recently, robotic assisted laparoscopy has been used in endometriosis surgery. There is still not enough evidence to support its routine use. It can help less experienced surgeons to do more minimally invasive surgery due to the shorter learning curve but it has its own limitations compared to conventional laparoscopy in terms of longer operation time and high cost.

\section{Acknowledgements}

None

\section{Conflict of Interest}

\section{None}

\section{References}

1. Burney R O, Giudice L C (2012) Pathogenesis and pathophysiology of endometriosis. Fertil Steril 98(3): 511-519.

2. Shaw R W, Soutter W P, Stanton S L (2003) Gynaecology. $3^{\text {rd }}$ Edn, Churchil Livingstone, London, p. 503.

3. Kovoor E, Nassif J, Miranda-Mendoza I, Wattiez A (2010) Endometriosis of bladder: outcomes after laparoscopic surgery. J Minim Invasive Gynecol 17(5): 600-604.

4. Channabasavaiah A D, Joseph J V (2010) Thoracic endometriosis: revisiting the association between clinical presentation and thoracic pathology based on thoracoscopic findings in 110 patients. Medicine (Baltimore) 89(3): 183-188.

5. Meuleman C, Vandenabeele B, Fieuws S, Spiessens C, Timmerman, et al. (2009) High prevalence of endometriosis in infertile women with normal ovulation and normospermic partners. Fertil Steril 92(1): 68-74.

6. Nnoaham K E, Hummelshoj L, Webster P, d'Hooghe T, de Cicco Nardone F, et al. (2011) Impact of endometriosis on quality of life and work productivity: a multicenter study across ten countries. Fertil Steril 96(2): 366-373.

7. Sepulcri R D P, Amaral V F D (2009) Depressive symptoms, anxiety, and quality of life in women with pelvic endometriosis. Eur J Obstet Gynecol Reprod Biol 142(1): 53-56.

8. Prasannan-Nair C, Manias T, Mathur R (2011) Management of endometriosis-related subfertility. The Obstetrician \& Gynaecologist 13(1): 1-6.
9. Hudelist G, Fritzer N, Thomas A, Niehues C, Opelt P, et al. (2012) Diagnostic delay for endometriosis in Austria and Germany: causes and possible consequences. Hum Reprod 27(12): 3412-3416.

10. Ballard K, Lowton K, Wright J (2006) What's the delay? A qualitative study of women's experiences of reaching a diagnosis of endometriosis. Fertil Steril 86(5): 1296-1301.

11. Kenney N, English J (2007) Surgical management of endometriosis. The Obstetrician \& Gynaecologist, 9 (3): 147-152.

12. Dunselman GA, Vermeulen N, Becker C, Calhaz-Jorge C, D’Hoogher T, et al. (2014) ESHRE guideline: management of women with endometriosis. Hum Reprod 29(3): 400-412.

13. Wykes C B, Clark T J, Khan K S (2004) Review: Accuracy of laparoscopy in the diagnosis of endometriosis: a systematic quantitative review. BJOG: An International Journal of Obstetrics \& Gynaecology 111(11): 1204-1212.

14. Duffy JMN, Arambage K, Correa FJS, Olive D, Farquhar C, et al. (2014) Laparoscopic surgery for endometriosis. Cochrane Database Syst Rev $3(4)$.

15. Wright J, Lotfallah $\mathrm{H}$, Jones $\mathrm{K}$, Lovel (2005) A randomized trial of excision versus ablation for mild endometriosis. Fertility and sterility 83(6): 1830-1836.

16. Healey M, Ang W, Cheng C (2010) Surgical treatment of endometriosis: a prospective randomized double-blinded trial comparing excision and ablation. Fertil Steril 94(7): 2536-2540.

17. Abbott J, Hawe J, Hunter D, Holmes M, Finn P, (2004) Laparoscopic excision of endometriosis: a randomized, placebo-controlled trial. Fertil Steril 82(4): 878-884.

18. Daniell JF, Lalonde CJ (1995) Advanced laparoscopic procedures for pelvic pain and dysmenorrhoea. Baillieres Clin Obstet Gynaecol 9(4): 795-808.

19. Proctor M L, Latthe P M, Farquhar C M, Khan K S, Johnson N P, et al. (2005) Surgical interruption of pelvic nerve pathways for primary and secondary dysmenorrhoea. Cochrane Database Syst Rev 19(4).

20.Sampson JA (1927) Peritoneal endometriosis due to menstrual dissemination of endometrial tissue into the peritoneal cavity. American Journal of Obstetrics and Gynecology 14(4): 422-469.

21. Nisolle M, Donnez J (1997) Peritoneal endometriosis, ovarian endometriosis, and adenomyotic nodules of the rectovaginal septum are three different entities. Fertil Steril 68(4): 585-596.

22. Farquhar C, Sutton C (1998) The evidence for the management of endometriosis. Curr Opin Obstet Gynecol 10(4): 321-332.

23. Luciano AA, Lowney J, Jacobs SL (1992) Endoscopic treatment of endometriosis-associated infertility. Therapeutic, economic and social benefits. J Reprod Med 37(7): 573-576.

24. Hart RJ, Hickey M,Maouris P, Buckett W (2008) Excisional surgery versus ablative surgery for ovarian endometriomata. Cochrane Database Syst Rev 16(2).

25. Carmona F, Martínez-Zamora M, Rabanal A, Martínez-Román S, et al. (2011) Ovarian cystectomy versus laser vaporization in the treatment of ovarian endometriomas: a Randomized clinical trial with a five-year follow-up. Fertil Steril 96(1): 251-254.

26. Meuleman C, Tomassetti C, D’Hoore A, Van Cleynenbreugel B, Penninckx F, et al. (2011) Surgical treatment of deeply infiltrating endometriosis with colorectal involvement. Hum Reprod Update 17(3): 311-326.

27. Donnez J, Squifflet J (2010) Complications, pregnancy and recurrence in a prospective series of 500 patients operated on by the shaving technique for deep rectovaginal endometriotic nodules. Hum Reprod 25(8): 1949-1958. 
28. De Cicco C, Corona R, Schonman R, Mailova K, Ussia A, et al. (2011) Bowe resection for deep endometriosis: a systematic review. An International Journal of Obstetrics \& Gynaecology 118(3): 285-291.

29. Daraï E, Dubernard G, Coutant C, Frey C, Rouzier R, et al. (2010) Randomized trial of laparoscopically assisted versus open colorectal resection for endometriosis: morbidity, symptoms, quality of life, and fertility. Ann Surg 251(6): 1018-1023.

30. Martin D C (2006) Hysterectomy for treatment of pain associated with endometriosis. J Minim Invasive Gynecol 13(6): 566-572.

31. Chalermchockchareonkit A, Tekasakul P, Chaisilwattan P, Sirimai K, Wahab N, et al. (2012) Laparoscopic hysterectomy versus abdominal hysterectomy for severe pelvic endometriosis. Int J Gynaecol Obstet 116(2): 109-111.
32. Carvalho L, Abrão M S, Deshpande A, Falcone T (2012) Robotics as a new surgical minimally invasive aproach to treatment of endometriosis: a systematic review. Int J Med Robot 8(2): 160-165.

33. Nezhat C R, Stevens A, Balassiano E, Soliemannjad R (2015) RoboticAssisted Laparoscopy vs Conventional Laparoscopy for the Treatment of Advanced Stage Endometriosis. J Minim Invasive Gynecol 22(1): 40-44.

34. Soto E, Lo Y, Friedman K, Soto C, Nezhat F, et al. (2011) Total laparoscopic hysterectomy versus da Vinci robotic hysterectomy: is using the robot beneficial? J Gynecol Oncol 22(4): 253-259. 